\title{
The Economic Importance and Social Values of Traditional Aquaculture in Rural Sierra Leone
}

\author{
Salieu Kabba Sankoh \\ Institute of Marine Biology and Oceanography, Fourah Bay College, University of Sierra Leone, Mount Aureol, Freetown, Sierra \\ Leone
}

\begin{abstract}
The economic viability and social value of traditional aquaculture in rural Sierra Leone were evaluated. The hypothesis tested was that traditional methods of farming fish in ponds were not economically viable, so farmers were investing their time/labour in less risky and more profitable livelihoods activities. The assumption in this assessment was that fish farming is an economic activity, and that for farmers to adopt or choose it over other livelihoods, the benefit-cost ratio of fish farming must be higher than that of alternative livelihoods. The main objective of the study was to assess why local farmers failed to adopt modern market oriented aquaculture production systems introduced by the Ministry of Fisheries and Marine Resources in rural Sierra Leone. Two hundred farmers owning fishponds on family lands were interviewed using semi-structured questionnaires and farmers' responses were analyzed using statistical tools (SPSS 16.0). The mean contributions of different livelihoods to total annual incomes of farmers and the social values they attached to these livelihoods were compared. Results of the study suggested that traditional fish farming was economically viable across the range of pond sizes sampled; but the medium-sized ponds were more profitable than the smallest and largest ponds, and labour accounted for $70 \%$ of total annual production costs in the systems assessed. The average benefit-cost ratio of 2.3 for fish farming was favorable compared with that of 0.83 for rice farming, which was ranked as most popular livelihood activity by farmers. The payback period for traditional aquaculture investments was less than five years using informal investment loans (without interest), and was favorably compared with that for tree crops farming, which was also popular among farmers. Additional benefits of fish farming included social capital and maintenance of kinship relationships.
\end{abstract}

Key words: Traditional aquaculture, fishponds, rural livelihoods, economic viability, benefit-cost ratio, payback period.

\section{Introduction}

To realize the full potential of aquaculture development in Sierra Leone, aquaculture production should be technically feasible, economically viable and socially acceptable. In addition, production methods used should be environmentally sustainable. Commercial fish farmers often consider profitability as the most important criteria in their decision to engage in fish farming [1, 2]. Poor rural farmers on the other hand try to minimise the risk by employing “portfolio" principles to spread risks among many enterprises [3]. Rural fish farmers may choose to: (1) produce fish primarily for household consumption (subsistence); (2) produce fish as a “cash crop” to

Corresponding author: Salieu Kabba Sankoh, Ph.D., research fields: sustainable aquaculture and aquatic resources management. E-mail: salieusankoh@hotmail.com. generate income; (3) produce fish for both household consumption and income generation.

They generally have little capital to invest and may not be aware of the amount of resources (in terms of time and labour) which they invest in fish farming. Commercial fish farmers on the other hand must know how much they spend and earn from their enterprises and how to work hard to maximize profit.

\subsection{Economic Flows in Rural Households in Sierra Leone}

Agricultural production was the most important economic activity in rural Sierra Leone. Most of the products were consumed at the household level and the surplus was marketed (sold in cash or bartered). In these production systems, the household members supply most of the inputs in the form of family labour, 
home-made implements, saved seeds and "bartered" inputs within the community. A few of the inputs may be obtained from outside the village using proceeds from the sale of the agriculture products. The main need for cash income is for schooling, health care and others, like clothing, weddings and circumcision ceremonies.

Marketing of surplus production was through resident or visiting village traders and nearby weekly markets, but village traders pay very low prices to farmers because they have few alternative outlets and only limited knowledge of prices outside their communities. The informal labour market was fairly developed, and remittances from relatives/friends in the cities and from abroad together with some newly emerging micro financing institutions were supporting new forms of market interactions within and outside the villages. A schematic representation of the basic economic flows in the rural economy of Sierra Leone is presented in Fig. 1.

\subsection{Objectives and Premise for the Economic} Evaluation of Traditional Fish Farming

The general objective of the study was to assess why local farmers failed to adopt modern market oriented aquaculture production systems introduced by the Ministry of Fisheries and Marine Resources in rural Sierra Leone. Specifically, the study aimed to: (1) generate information on the economics of fish farming under current technology in inland valley swamps of four districts in Sierra Leone; (2) estimate total annual fish production and marketable surplus in the four districts; (3) assess the economic and social value of fish farming relative to that of other livelihoods activities in the study areas.

The hypothesis tested was that traditional methods of farming fish in ponds were not economically viable and rural farmers were therefore investing their time/labour in less risky and more profitable livelihoods activities. The assessment was carried out with the assumption that fish farming is an economic activity, and that for farmers to adopt or choose modern methods of fish farming over other livelihoods, the benefit-cost ratio of fish farming must be higher than that of alternative livelihoods.

The premise for this assessment was that rural farmers would only adopt modern methods of fish farming, if they perceive that it offered more benefits

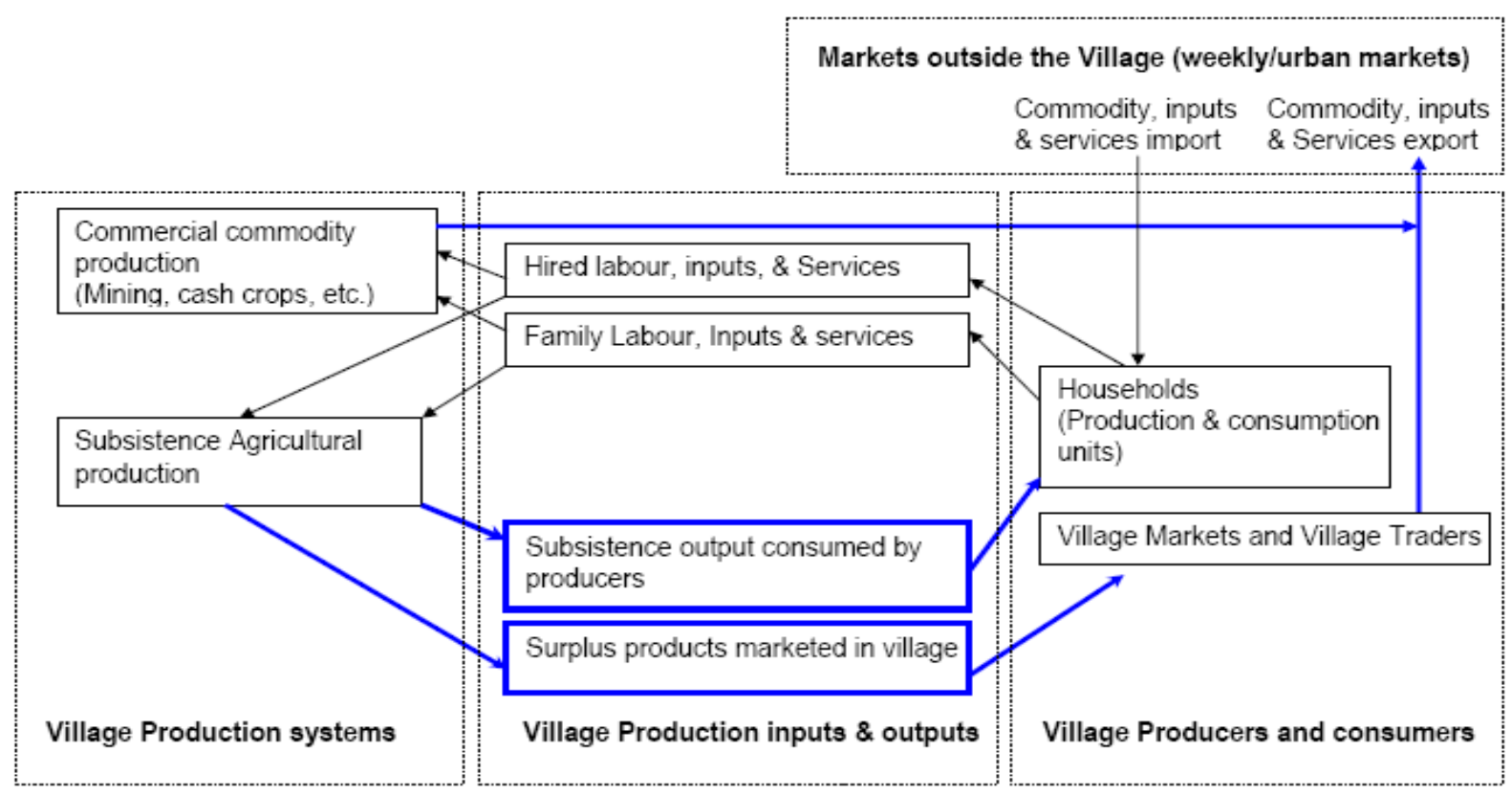

Fig. 1 Basic economic flows in rural Sierra Leone reflecting an intermediate level of economic development.

Source: adapted from Taylor and Adelman [4]. Commercial products were exported to markets outside the village and some basic commodities and inputs were imported from the same markets. 
over alternative activities. The study therefore assessed the relative contribution of fish farming and other livelihoods activities to farmers' incomes and the benefit-cost ratio of fish culture in ponds. All tangible benefits and costs of fish farming and other livelihoods were expressed in monetary terms to enhance comparison of the economic value of fish farming relative to other rural livelihoods in Sierra Leone.

\section{Methods}

\subsection{Data Collection Methods}

Two hundred semi-structured questionnaires were administered to farmers who had one or more ponds on family or privately owned (inherited) land. In Sierra Leone, rural farmers do not own land by deed, plots of land are temporarily allocated to family members by family heads or traditional authorities [5]. Respondents were selected randomly from a national aquaculture baseline study database [6]; most were head of households with a mean age of 48 years and most (82\%) were male. Data analysis and economic assumptions used in the analytical process are described below. Statistical analysis was done using SPSS version 16.0.

2.2 Ranking of Livelihoods and Estimation of the Mean Contributions of the Different Livelihoods to Farmers'Annual Incomes

Two hundred Farmers were asked to rank all their livelihoods (individually) according to the importance to them. The three most important livelihoods of all farmers were then pooled together to determine the predominant and most popular livelihoods. The mean contributions of the different livelihoods to farmers' annual incomes were calculated and compared to farmers ranking criteria results.

\subsection{Estimation of the Costs of and Returns from Fish}

\section{Production}

The costs incurred by fish farmers in producing, processing, transporting and marketing fish were grouped as follows:

(1) Pre-stocking costs (cost of land, tools/equipments and labour for pond construction);

(2) Stocking, feeding and general management costs (cost of obtaining and transporting fingerlings to the pond sites, cost of feed and manure, cost of labour for brushing around the ponds and sometimes constructing a fence around the pond);

(3) Harvesting and marketing costs (labour cost for harvesting the fish, cost of fishing gear for harvesting the fish, cost of processing, transporting and marketing the fish).

Economic indicators measured/assessed in this study included the projected costs of fish production, gross incomes, net incomes, benefit-cost ratios and payback period $^{1}$; the net present value ${ }^{2}$ (NPV) of future cash flow (base year 2004) with an average discount rate of $20 \%$ took into account the high inflation rates and high risk investment nature of traditional fish farming (low risk investment in treasury bearer bonds yielded between $20 \%$ and $30 \%$ per annum in 2004) [7]. The costs, useful life and salvage value (where applicable) of tool and equipment used by farmers were all factored in the analysis. In cases where actual market values of input items were not available/known, the opportunity cost was used to derive inputted values.

\section{Results}

\subsection{Ranking of Livelihood Activities by Farmers}

The overall rankings of the three most important livelihoods activities by the 200 farmers sampled showed that rice farming was the most popular activities among rural communities in Sierra Leone. Other popular livelihoods activities were oil palm plantations, root tubers (cassava and potatoes), wild fishing/fish farming, ground nuts farming, formal employment, livestock rearing, cash crops (fruits

\footnotetext{
${ }^{1}$ http://www.valuebasedmanagement.net/methods_payback_ period.html.

${ }^{2}$ http://www.investopedia.com/terms/n/npv.asp.
} 
including plantain and banana), cash crops (cocoa and coffee), vegetable gardening, pineapple garden and trading. In total $42.5 \%$ of all farmers interviewed ranked rice farming among their three most important livelihoods (Table 1). Swamp rice farming was more popular in the North than in the South, while coffee/cocoa plantations and mining were not recorded in the North. Fish farming was not at the top of farmers' activities lists in the study areas.

\subsection{Mean Contributions of the Three Most Important} Livelihoods to Annual Incomes of Farmers in Rural Sierra Leone

The percentage contributions of the three most important livelihoods and the mean value (\$) of reported production levels were presented in Tables 1 and 2. Overall, swamp and upland rice farming contributed $82.8 \%$ and $68.5 \%$ to farmers' total average annual incomes, respectively.

Money-wise, livestock rearing, cash crop production and formal employment were most rewarding, with average income exceeding \$300 per year. Income from rice farming was around $\$ 150$ per year and fish farming was $\$ 140$. Farmers earn just under \$200 from their three most important livelihoods. If one considers that $85 \%$ of farmers' total annual income comes from their three most important livelihoods, one can conclude that farmers in rural Sierra Leone are mostly poor with average annual incomes of less than $\$ 300$.

Differences in the percentage contributions of the three most important livelihoods activities in the North and South were not significant $(P>0.05)$, but the mean value of production levels (in $\$$ ) for the North and South were significant $(P<0.05)$ (Table 2). There was a very strong positive correlation between the mean contributions of primary activities and the total annual incomes of farmers $(R=0.849)$ (Fig. 2).

\subsection{Farmers' Motivations for Primary Livelihood Activities and Fish Farming}

Farmers' motives for undertaking livelihoods activities (including fish farming) were food security and income generation (Table 3). However, the food security motives dominated in comparison with the income generation motives, because the five most popular activities were not the most rewarding in terms of cash value (in \$) of the production level reported (Table 2). Only one farmer grew fish for making money and two for academic reasons.

\subsection{Costs of and Returns from Fish Production in} Ponds by Farm Sizes Categories and Region

The mean annual costs of and returns from fish farming in fish ponds of various sizes in the two regions are presented in Tables 4 and 5, and the projected costs and returns from fish farming for 10 years of continuous production at the same level for different farm sizes and regions are presented in Tables 5 and 6, respectively.

\section{Discussion}

4.1 Relative Importance of Different Livelihoods Activities to Farmers

Farmers had a logical ranking system of their different livelihoods activities. Generally, the relative importance of livelihood activities was based on a combination of the following criteria:

(1) Importance of rice as staple food: rice is the staple food in Sierra Leone and most people would consider themselves as starving without rice. Annual per capita consumption of rice (104 kg) in Sierra Leone is among the highest in Sub-Saharan Africa and rural farmers only define themselves as farmers if they grow rice [8];

(2) Income derived from the activities: some crops were grown entirely for cash, for example cocoa and coffee; others are grown mostly for cash, e.g., ground nuts and oil palm. Farmers whose motives were predominantly for income generation therefore regarded cash crops production as their primary livelihoods, whereas those whose primary motive for farming was for food security regarded rice farming as their primary livelihood [9]; 
Table 1 Livelihoods importance rankings and mean contributions (\%) of the three most important livelihoods activities to the annual incomes of all 200 farmers $(n=115$ farmers in the North and $n=85$ farmers in the South).

\begin{tabular}{|c|c|c|c|c|c|c|c|c|c|c|c|c|c|c|c|c|}
\hline \multirow[t]{2}{*}{ Rank } & \multirow[t]{2}{*}{ livelihoods activities } & \multicolumn{3}{|c|}{$\begin{array}{l}\text { No. of all farmers engaged in the } \\
\text { different activities }\end{array}$} & \multicolumn{3}{|c|}{$\begin{array}{l}\text { Contribution of activity to } \\
\text { annual income as primary } \\
\text { livelihoods }\end{array}$} & \multicolumn{3}{|c|}{$\begin{array}{l}\text { Contribution of activity to } \\
\text { annual income as secondary } \\
\text { livelihoods } \\
\end{array}$} & \multicolumn{3}{|c|}{$\begin{array}{l}\text { Contribution of activity to } \\
\text { annual income as tertiary } \\
\text { livelihoods }\end{array}$} & \multicolumn{3}{|c|}{$\begin{array}{l}\text { Total contributions of three } \\
\text { most important (\%) }\end{array}$} \\
\hline & & Primary & Second & Tertiary & $\begin{array}{l}\text { All } \\
(\%)\end{array}$ & $\begin{array}{l}\text { North } \\
(\%)\end{array}$ & $\begin{array}{l}\begin{array}{l}\text { South } \\
(\%)\end{array} \\
\end{array}$ & $\begin{array}{l}\text { All } \\
(\%)\end{array}$ & $\begin{array}{l}\text { North } \\
(\%)\end{array}$ & $\begin{array}{l}\begin{array}{l}\text { South } \\
(\%)\end{array} \\
\end{array}$ & $\begin{array}{l}\text { All } \\
(\%)\end{array}$ & $\begin{array}{l}\text { North } \\
(\%)\end{array}$ & $\begin{array}{l}\begin{array}{l}\text { South } \\
(\%)\end{array} \\
\end{array}$ & $\begin{array}{l}\text { All } \\
(\%)\end{array}$ & $\begin{array}{l}\text { North } \\
\text { (\%) }\end{array}$ & $\begin{array}{l}\text { South } \\
(\%)\end{array}$ \\
\hline 1 & Swamp rice farming & 96 & 47 & 13 & 46.8 & 46.6 & 48.6 & 20.4 & 23.6 & 18.1 & 15.6 & 20.0 & 14.8 & 82.8 & 90.2 & 81.5 \\
\hline 2 & Upland rice farming & 33 & 53 & 12 & 33.3 & 39.2 & 30.7 & 23.1 & 24.1 & 18.6 & 12.1 & & & 68.5 & 63.3 & 49.3 \\
\hline 3 & Oil palm plantation & 31 & 34 & 13 & 50.7 & 48.0 & 51.4 & 26.5 & 28.8 & 23.9 & 16.0 & 14.4 & 22.5 & 93.2 & 91.2 & 97.8 \\
\hline 4 & $\begin{array}{l}\text { Root tubers } \\
\text { (cassava \& potatoes) }\end{array}$ & 3 & 12 & 46 & 60.0 & 60.0 & - & 18.8 & 18.6 & 20.0 & 13.3 & 15.0 & 12.8 & 92.1 & 93.6 & 32.8 \\
\hline 5 & Fish farming & 5 & 11 & 39 & 46.7 & 50.0 & 40.0 & 22.2 & 24.3 & 15.0 & 13.9 & 16.5 & 8.6 & 82.8 & 90.8 & 63.6 \\
\hline 6 & $\begin{array}{l}\text { Ground nuts } \\
\text { production }\end{array}$ & 1 & 6 & 20 & 35.0 & & 35.0 & 24.0 & 35.0 & 21.3 & 11.3 & 11.5 & 11.0 & 70.3 & 46.5 & 67.3 \\
\hline 7 & Formal employment & 20 & 0 & 1 & 52.2 & 50.0 & 52.5 & - & - & - & 15.0 & - & 15.0 & 67.2 & 50.0 & 67.5 \\
\hline 8 & Livestock rearing & 1 & 1 & 14 & 60.0 & 60.0 & & & & & 14.6 & 15.8 & 13.8 & 74.6 & 75.8 & 13.8 \\
\hline 9 & $\begin{array}{l}\text { Cash crops } \\
\text { (plantain/banana) }\end{array}$ & 1 & 10 & 4 & 60.0 & 50.0 & & 18.8 & 22.5 & 11.5 & 13.8 & 15.0 & 12.5 & 92.6 & 87.5 & 24.0 \\
\hline 10 & $\begin{array}{l}\text { Cash crops } \\
\text { (cocoa \& coffee) }\end{array}$ & 2 & 7 & 7 & 60.0 & - & 60.0 & 14.0 & - & 14.0 & 12.1 & - & 12.1 & 86.1 & - & 86.1 \\
\hline 11 & Vegetable gardening & 2 & 6 & 7 & 32.5 & & 32.5 & 21.3 & 20.0 & 21.7 & 12.9 & 10.0 & 15.0 & 66.7 & 30.0 & 69.2 \\
\hline 12 & Wild fishing & 0 & 5 & 7 & & & & 23.0 & 22.5 & 25.0 & 15.7 & 15.7 & & 38.7 & 38.2 & 25.0 \\
\hline 13 & Pineapple garden & 0 & 5 & 5 & 50.0 & & & 20.0 & & 20.0 & 12.0 & 12.0 & & 82.0 & 12.0 & 20.0 \\
\hline 14 & Trading & 2 & 2 & 4 & 45.0 & 45.0 & & & & & 20.0 & & & 65.0 & 45.0 & - \\
\hline 15 & Palm wine tapping & 1 & 0 & 1 & 30.0 & - & 30.0 & - & - & - & 20.0 & 20.0 & - & 50.0 & 20.0 & 30.0 \\
\hline 16 & Casual employment & 0 & 0 & 2 & - & - & - & - & - & - & 10.0 & 10.0 & 10.0 & 10.0 & 10.0 & 10.0 \\
\hline 17 & Construction worker & 0 & 0 & 2 & & & & & & & 12.5 & 12.5 & & 12.5 & 12.5 & - \\
\hline 18 & Mining & 1 & - & - & 80.0 & - & 80.0 & - & - & - & - & - & - & 80.0 & - & 80.0 \\
\hline 19 & Tailor & 0 & 1 & 0 & - & - & - & 30.0 & - & 30.0 & - & 20.0 & - & 30.0 & 20.0 & 30.0 \\
\hline \multicolumn{2}{|c|}{ Average } & 200 & 200 & 197 & 49.5 & 49.9 & 46.1 & 21.8 & 24.4 & 19.9 & 14.2 & 14.9 & 13.5 & 85.0 & 89.0 & 79.0 \\
\hline
\end{tabular}


Table 2 Mean value of production levels reported for the three most important livelihoods activities reported by 200 farmers $(n=115$ in the North and $n=85$ in the South).

\begin{tabular}{|c|c|c|c|c|c|c|c|c|c|c|c|c|c|c|c|c|}
\hline \multirow[t]{2}{*}{ Livelihoods activities } & \multicolumn{3}{|c|}{$\begin{array}{c}\text { No. of farmers engaged in } \\
\text { activity }\end{array}$} & \multirow{2}{*}{$\begin{array}{l}\text { Livelihood } \\
\text { importance } \\
\text { ratings/ } \\
\text {-popularity }\end{array}$} & \multicolumn{3}{|c|}{$\begin{array}{l}\text { Mean values of the } \\
\text { contributions of primary } \\
\text { livelihoods (\$) }\end{array}$} & \multicolumn{3}{|c|}{$\begin{array}{l}\text { Mean values of the } \\
\text { contributions of secondary } \\
\text { (\$) }\end{array}$} & \multicolumn{3}{|c|}{$\begin{array}{l}\text { Mean values of the } \\
\text { contributions of tertiary } \\
\text { livelihoods (\$) }\end{array}$} & \multicolumn{3}{|c|}{$\begin{array}{c}\text { Total values of } \\
\text { contributions of the three } \\
\text { most important livelihoods } \\
(\$)\end{array}$} \\
\hline & Primary & Secondary & Tertiary & & All & North & South & All & North & South & All & North & South & All & North & South \\
\hline Swamp rice farming & 96 & 47 & 13 & 1 & 77 & 75 & 103 & 45 & 51 & 41 & 41 & - & 41 & 163 & 126 & 185 \\
\hline Upland rice farming & 33 & 53 & 12 & 2 & 55 & 68 & 49 & 41 & 40 & 48 & 33 & 59 & 30 & 129 & 167 & 127 \\
\hline Oil palm plantation & 31 & 34 & 13 & 3 & 115 & 72 & 125 & 66 & 46 & 88 & 0 & 32 & 38 & 181 & 150 & 251 \\
\hline $\begin{array}{l}\text { Root tubers } \\
\text { (cassava \& potatoes) }\end{array}$ & 3 & 12 & 46 & 4 & 155 & 155 & 0 & 30 & 21 & 93 & 24 & 20 & 39 & 209 & 196 & 132 \\
\hline Fish farming & 5 & 11 & 39 & 5 & 64 & 61 & 71 & 46 & 40 & 70 & 31 & 35 & 23 & 141 & 136 & 164 \\
\hline Ground nuts production & 1 & 6 & 20 & 6 & 65 & 0 & 65 & 48 & 91 & 38 & 19 & 15 & 22 & 132 & 106 & 125 \\
\hline Formal employment & 20 & 0 & 1 & 7 & 230 & 204 & 233 & 0 & 0 & 0 & 83 & 0 & 83 & 313 & 204 & 316 \\
\hline Livestock rearing & 1 & 1 & 14 & 8 & 289 & 289 & 0 & 0 & 0 & 0 & 35 & 32 & 38 & 324 & 321 & 38 \\
\hline $\begin{array}{l}\text { Cash crops } \\
\text { (fruit trees including } \\
\text { plantain/banana) }\end{array}$ & 1 & 10 & 4 & 9 & 241 & 241 & 0 & 27 & 34 & 11 & 43 & 21 & 89 & 311 & 296 & 100 \\
\hline $\begin{array}{l}\text { Cash crops } \\
\text { (cocoa \& coffee) }\end{array}$ & 2 & 7 & 7 & 10 & 355 & 0 & 355 & 35 & 0 & 35 & 23 & 0 & 23 & 413 & & 413 \\
\hline Vegetable gardening & 2 & 6 & 7 & 11 & 76 & 0 & 76 & 86 & 89 & 84 & 10 & 10 & 0 & 172 & 99 & 160 \\
\hline Wild fishing & 0 & 5 & 7 & 12 & 0 & 0 & 0 & 26 & 0 & 44 & 26 & 26 & 0 & 52 & 26 & 44 \\
\hline Pineapple garden & 0 & 5 & 5 & 13 & 0 & 0 & 0 & 83 & 0 & 83 & 12 & 12 & 0 & 95 & 12 & 83 \\
\hline Trading & 2 & 2 & 4 & 14 & 81 & 81 & 0 & 0 & 0 & 0 & 0 & 0 & 0 & 81 & 81 & \\
\hline Palm wine tapping & 1 & 0 & 1 & 15 & 78 & 0 & 78 & 0 & 0 & 0 & 15 & 15 & 0 & 93 & 15 & 78 \\
\hline Casual employment & 0 & 0 & 2 & 16 & 0 & 0 & 0 & 0 & 0 & 0 & 31 & 0 & 32 & 31 & & 32 \\
\hline Construction worker & 0 & 0 & 2 & 17 & 0 & 0 & 0 & 0 & 0 & 0 & 18 & 18 & 0 & 18 & 18 & \\
\hline Mining & 1 & 0 & 0 & 18 & 666 & 0 & 666 & 0 & 0 & 0 & 0 & 0 & 0 & 666 & & 666 \\
\hline Tailor & 0 & 1 & 0 & 19 & 0 & 0 & 0 & 33 & 33 & 0 & 0 & 0 & 0 & 33 & 33 & \\
\hline Average & 200 & 200 & 197 & & 182 & 138 & 182 & 47 & 50 & 58 & 30 & 24 & 41 & 187 & 124 & 182 \\
\hline
\end{tabular}

The average official exchange rates (Leone/\$) was Leone2,701.30 = \$1; popularity rating: 1 = most popular. 


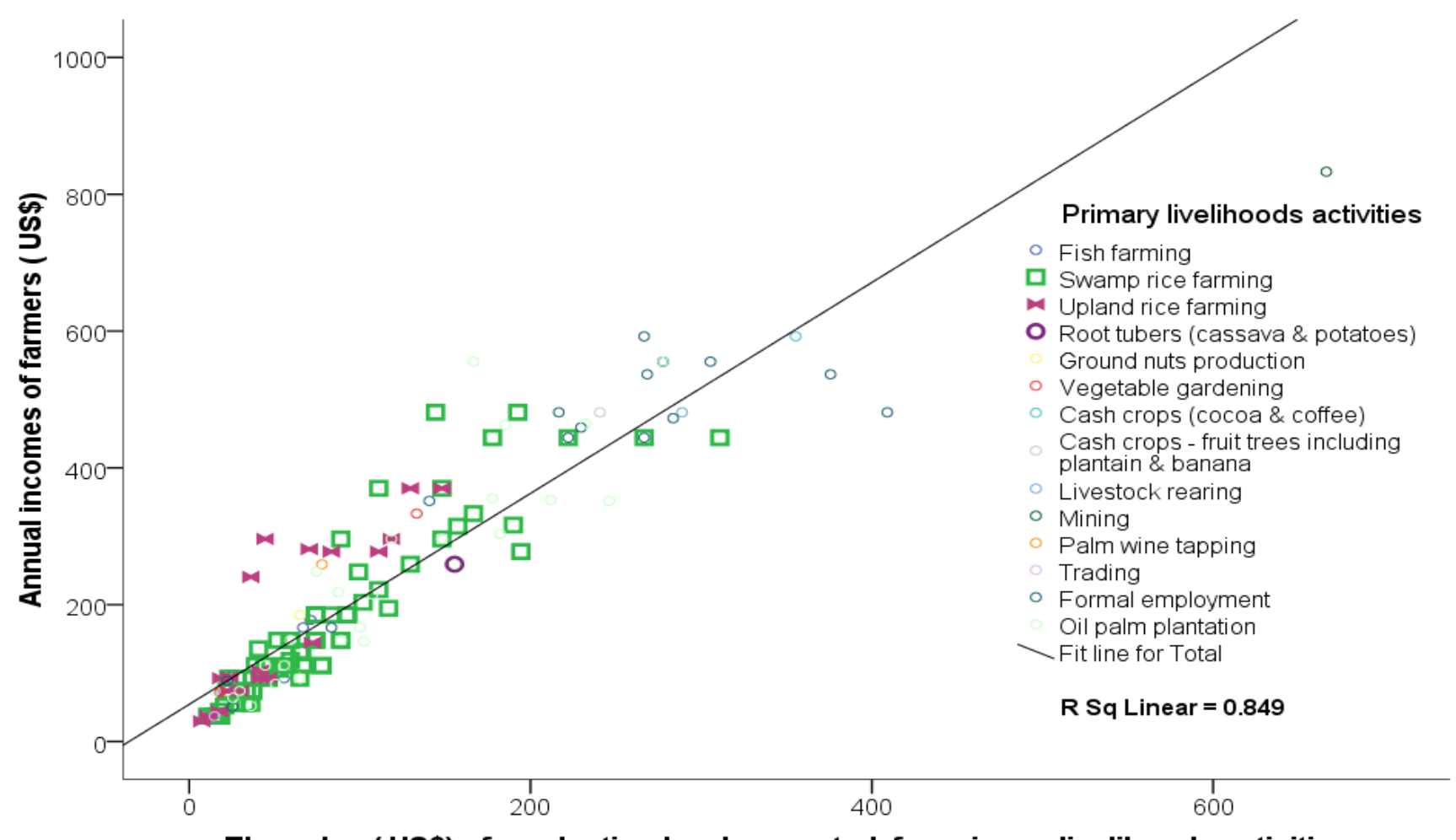

Fig. 2 Correlation between estimated amount money derived from primary livelihood activities and total annual incomes of farmers.

Table 3 Motivation for primary livelihoods, three most important livelihoods and fish farming.

\begin{tabular}{|c|c|c|c|c|c|c|}
\hline \multirow{2}{*}{ Motivation } & \multicolumn{2}{|c|}{ Primary livelihood } & \multicolumn{2}{|c|}{ First three livelihoods } & \multicolumn{2}{|c|}{ Fish farming } \\
\hline & $N$ & $\%$ & $N R$ & $\%$ & $N$ & $\%$ \\
\hline Food security and income generation & 156 & 78 & 480 & 81.2 & 182 & 91 \\
\hline Income generation only & 36 & 18 & 94 & 15.9 & 1 & 0.5 \\
\hline Food security only & 8 & 4 & 17 & 2.9 & 15 & 7.5 \\
\hline Academic & 0 & 0 & 0 & 0 & 2 & 1 \\
\hline Total & 200 & 100 & 591 & 100 & 200 & 100 \\
\hline
\end{tabular}

$N=$ number of farmers, $N R=$ total number of responses, \% = percentage of total number of farmers (200) or percentage of total responses (591). 
Table 4 Average costs and returns from fish farming in the first year of production and the percentage contributions of the different inputs costs to the total production cost by farm size category.

\begin{tabular}{|c|c|c|c|c|c|c|c|c|}
\hline Cost/returns item & $\begin{array}{l}\text { Small farms } \\
(N=138, \\
\left.S=276.8 \pm 0.1 \mathrm{~m}^{2}\right)\end{array}$ & $\%$ & $\begin{array}{l}\text { Medium farms } \\
(N=15, \\
\left.S=620.5 \pm 16.4 \mathrm{~m}^{2}\right)\end{array}$ & $\%$ & $\begin{array}{l}\text { Large farms } \\
(N=138 \\
\left.S=1,903.7 \pm 23.2 \mathrm{~m}^{2}\right)\end{array}$ & $\%$ & $\begin{array}{l}\text { All farms } \\
(N=15, \\
\left.S=479.6 \pm 35.7 \mathrm{~m}^{2}\right)\end{array}$ & $\%$ \\
\hline \multicolumn{9}{|l|}{ Cost } \\
\hline Total cost of land (\$/ha/year) & $328 \pm 12$ & 10 & $328 \pm 9$ & 14 & $327 \pm 39$ & 19 & $327 \pm 24$ & 13 \\
\hline Total cost of tools (\$/ha/year) & $78 \pm 5$ & 2 & $81 \pm 6$ & 4 & $42 \pm 8$ & 2 & $68 \pm 4$ & 3 \\
\hline Total cost of the 1 st year labour ( $\$ /$ ha/year) & $2,635 \pm 162$ & 77 & $1,457 \pm 55$ & 64 & $1,028 \pm 14$ & 60 & $1,798 \pm 91$ & 70 \\
\hline Total cost of fingerlings ( $\$ /$ ha/year) & $267 \pm 26$ & 8 & $336 \pm 39$ & 15 & $284 \pm 5$ & 16 & $291 \pm 30$ & 11 \\
\hline Total cost of feed, manure/fertilizer ( $\$ /$ ha/year) & $41 \pm 2$ & 1 & $20 \pm 1$ & 1 & $10 \pm 3$ & 1 & $26 \pm 1$ & 1 \\
\hline Total cost of fishing gear (\$/ha/year) & $51 \pm 2$ & 2 & $35 \pm 3$ & 2 & $28 \pm 2$ & 2 & $39 \pm 2$ & 2 \\
\hline Total cost of wood, ropes \& thatch ( $\$ /$ ha/year) & $9 \pm 0$ & 0 & $6 \pm 0$ & 0 & $5 \pm 0$ & 0 & $7 \pm 0$ & 0 \\
\hline \multicolumn{9}{|l|}{ Returns } \\
\hline Money from requested sales (\$/ha/year) & $913 \pm 54$ & 19 & $668 \pm 71$ & 17 & $326 \pm 37$ & 17 & $665 \pm 36$ & 18 \\
\hline Money from sale of fish at main harvest (\$/ha/year) & $2,127 \pm 92$ & 44 & $1,697 \pm 165$ & 44 & $832 \pm 115$ & 43 & $1,603 \pm 83$ & 44 \\
\hline Money from sale of fingerlings ( $\$ /$ ha/year) & $216 \pm 44$ & 4 & $103 \pm 19$ & 3 & $48 \pm 21$ & 2 & $133 \pm 20$ & 4 \\
\hline Money from farmer’s own consumption (\$/ha/year) & $1,297 \pm 74$ & 27 & $1,043 \pm 123$ & 27 & $492 \pm 87$ & 25 & $982 \pm 61$ & 27 \\
\hline Money from fingerlings used by farmer ( $\$ /$ ha/year) & $286 \pm 27$ & 6 & $342 \pm 47$ & 9 & $247 \pm 57$ & 13 & $290 \pm 30$ & 8 \\
\hline
\end{tabular}

$S=$ mean farm/pond size in $\mathrm{m}^{2}, N=$ number of respondents, all values rounded up to the nearest $\$$.

Table 5 Projected costs and returns from 10 years of continuous fish production at the same level by farm size category.

\begin{tabular}{|c|c|c|c|c|}
\hline $\begin{array}{l}\text { Projected cost and returns from fish farming using } \\
\text { ponds for 10years }\end{array}$ & $\begin{array}{l}\text { Small farms }(N=138, \\
\left.S=276.81 \pm 0.06 \mathrm{~m}^{2}\right)\end{array}$ & $\begin{array}{l}\text { Medium farmers }(N=47 \\
\left.S=620.53 \pm 16.42 \mathrm{~m}^{2}\right)\end{array}$ & $\begin{array}{l}\text { Large farms }(N=15, \\
\left.S=1,903.70 \pm 230.22 \mathrm{~m}^{2}\right)\end{array}$ & $\begin{array}{l}\text { All farms }(N=200 \\
\left.S=479.60 \pm 35.68 \mathrm{~m}^{2}\right)\end{array}$ \\
\hline \multicolumn{5}{|l|}{ Cost } \\
\hline The 1st year total production cost (\$/ha/year) & $3,410 \pm 208$ & $2,262 \pm 114$ & $1,723 \pm 244$ & $2,556 \pm 153$ \\
\hline Running cost in year 2 (\$/ha/year) & $1,272 \pm 41$ & $998 \pm 51$ & $707 \pm 67$ & $1,027 \pm 51$ \\
\hline 10 years discounted production cost $(\$ / \mathrm{ha})$ & $10,377 \pm 441$ & $7,757 \pm 411$ & $5,601 \pm 640$ & $8,187 \pm 443$ \\
\hline Mean annual production cost (\$/ha/year) & $1,038 \pm 44$ & $776 \pm 41$ & $560 \pm 64$ & $819 \pm 44$ \\
\hline \multicolumn{5}{|l|}{ Returns } \\
\hline The 1st year mean gross income $(\$ / \mathrm{ha})$ & $4,839 \pm 292$ & $3,854 \pm 424$ & $1,945 \pm 317$ & $3,673 \pm 229$ \\
\hline 10 years total discounted gross income $(\$ / \mathrm{ha})$ & $25,336 \pm 34$ & $19,888 \pm 2,102$ & $10,200 \pm 81$ & $19,169 \pm 1,063$ \\
\hline Gross income (\$/ha/year) & $2,534 \pm 124$ & $1,989 \pm 210$ & $1,020 \pm 144$ & $1,917 \pm 106$ \\
\hline Net profit (\$/ha/year) & $1,496 \pm 80$ & $1,213 \pm 169$ & $460 \pm 80$ & $1,098 \pm 62$ \\
\hline Gross profit margin & 59.0 & 61.0 & 45.1 & 57.3 \\
\hline Benefit-Cost ratio & 2.4 & 2.6 & 1.8 & 2.3 \\
\hline Payback period (loans without interest) & 4.1 & 3.9 & 5.5 & 4.3 \\
\hline Payback period (loans with 20\% interest) & 6.6 & 6.2 & 9.6 & 6.9 \\
\hline
\end{tabular}

$S=$ mean farm/pond size in $\mathrm{m}^{2}, N=$ number of respondents, all values rounded up to the nearest $\$$. 
Table 6 Average cost and returns from fish farming in the first year of production and the percentage contributions of the different inputs cost to the total production cost by region.

\begin{tabular}{|c|c|c|c|c|c|c|}
\hline Cost/return item & $\begin{array}{l}\text { Northern region } \pm \mathrm{SE} \\
\left(N=115, S=375.03 \mathrm{~m}^{2}\right)\end{array}$ & $\%$ & $\begin{array}{l}\text { Southern region } \pm \mathrm{SE} \\
\left(N=85, S=621.07 \mathrm{~m}^{2}\right)\end{array}$ & $\%$ & $\begin{array}{l}\text { All } \pm \text { SE } \\
\left(N=200, S=479.6 \mathrm{~m}^{2}\right)\end{array}$ & $\%$ \\
\hline \multicolumn{7}{|l|}{ Cost } \\
\hline Total cost of land (\$/ha/year) & $328 \pm 20$ & 12 & $327 \pm 40$ & 13 & $327 \pm 24$ & 13 \\
\hline Total cost of tools per year (\$/ha/year) & $88 \pm 6$ & 3 & $52 \pm 6$ & 2 & $68 \pm 4$ & 3 \\
\hline Total cost of the 1st year labour ( $\$ /$ ha/year) & $1,883 \pm 83$ & 71 & $1,729 \pm 143$ & 68 & $1,798 \pm 91$ & 70 \\
\hline Total cost of fingerlings ( $\$ /$ ha/year) & $268 \pm 23$ & 10 & $382 \pm 68$ & 15 & $291 \pm 30$ & 11 \\
\hline Total cost of feed, manure/fertilizer ( $\$ /$ ha/year) & $31 \pm 1$ & 1 & $21 \pm 2$ & 1 & $26 \pm 1$ & 1 \\
\hline Total cost of fishing gear (\$/ha/year) & $45 \pm 2$ & 2 & $35 \pm 3$ & 1 & $39 \pm 2$ & 2 \\
\hline Total cost of wood, ropes and thatch (\$/ha/year) & $7 \pm 0$ & 0 & $6 \pm 1$ & 0 & $7 \pm 0$ & 0 \\
\hline \multicolumn{7}{|l|}{ Return } \\
\hline Money from requested sales (\$/ha/year) & $802 \pm 62$ & 19 & $550 \pm 42$ & 17 & $665 \pm 36$ & 18 \\
\hline Money sold at main harvest (\$/ha/year) & $1,863 \pm 125$ & 44 & $1,378 \pm 107$ & 42 & $1,603 \pm 83$ & 44 \\
\hline Money from sale of fingerlings ( $\$ /$ ha/year) & $163 \pm 31$ & 4 & $112 \pm 25$ & 3 & $133 \pm 20$ & 4 \\
\hline Money from farmer’s own consumption (\$/ha/year) & $1,162 \pm 94$ & 27 & $831 \pm 79$ & 26 & $982 \pm 61$ & 27 \\
\hline Money from fingerlings used by farmer (\$/ha/year) & $268 \pm 23$ & 6 & $380 \pm 68$ & 12 & $290 \pm 30$ & 8 \\
\hline
\end{tabular}

$S=$ mean farm/pond size in $\mathrm{m}^{2}, N=$ number of respondents, all values rounded up to the nearest $\$$.

Table 7 Projected costs of inputs and returns for 10 years continuous production at the same level by region.

\begin{tabular}{|c|c|c|c|}
\hline $\begin{array}{l}\text { Cost and returns from fish farming using ponds } \\
\text { for } 10 \text { years }\end{array}$ & $\begin{array}{l}\text { North } \\
\left(N=115, S=375.03 \mathrm{~m}^{2}\right)\end{array}$ & $\begin{array}{l}\text { South } \\
\left(N=85, S=621.07 \mathrm{~m}^{2}\right)\end{array}$ & $\begin{array}{l}\text { All } \\
\left(N=200, S=479.6 \mathrm{~m}^{2}\right)\end{array}$ \\
\hline \multicolumn{4}{|l|}{ Cost } \\
\hline The 1st year total production cost (\$/ha/year) & $2,649 \pm 135$ & $2,552 \pm 262$ & $2,556 \pm 153$ \\
\hline Running cost in the 2nd year ( $\$ /$ ha/year) & $1,189 \pm 55$ & $889 \pm 78$ & $1,027 \pm 51$ \\
\hline 10 years discounted production cost $(\$ / \mathrm{ha})$ & $9,152 \pm 448$ & $7,426 \pm 701$ & $8,187 \pm 443$ \\
\hline Mean annual cost of production (\$/ha/year) & $915 \pm 45$ & $743 \pm 70$ & $819 \pm 44$ \\
\hline \multicolumn{4}{|l|}{$\begin{array}{l}\text { Return } \\
\end{array}$} \\
\hline The 1st year mean gross income (\$/ha/year) & $4,258 \pm 334$ & $3,251 \pm 22$ & $3,673 \pm 229$ \\
\hline 10 years total discounted gross income $(\$ / \mathrm{ha})$ & $22,038 \pm 1,555$ & $16,882 \pm 1,142$ & $19,169 \pm 1,063$ \\
\hline Gross income (\$/ha/year) & $2,204 \pm 155$ & $1,688 \pm 114$ & $1,917 \pm 106$ \\
\hline Net profit (\$/ha/year) & $1,289 \pm 111$ & $946 \pm 44$ & $1,098 \pm 62$ \\
\hline Gross profit margin & 58.5 & 56.0 & 57.3 \\
\hline Benefit-Cost ration & 2.4 & 2.3 & 2.3 \\
\hline Payback time (years) & 4.2 & 4.4 & 4.3 \\
\hline Payback period on loans with $20 \%$ interest rate (years) & 6.8 & 7.1 & 6.9 \\
\hline
\end{tabular}

$S=$ mean farm/pond size in $\mathrm{m}^{2}, N=$ number of respondents, all values rounded up to the nearest $\$$. 
(3) Social security considerations: tree crops were grown by most farmers, because they keep on producing every year with little effort from the farmers. Tree crops including fruit trees, oil palm, cocoa, banana and plantain were all regarded as social security crops for farmers at old age [10];

(4) Synchronisation of harvest with hunger periods: some crops like cassava, millet and ground nuts were reported to be life saving crops during the hunger periods (July to September). Cassava was described as a hunger crop, because it could be eaten raw in the fields before final maturation, harvesting and cooking or processing into “foofoo”, “gari” or “Kondogbala” [11].

Traditional fish farming was fitted into the social security considerations of rural farmer's livelihoods ranking and could also fit into the hunger period synchronization livelihoods strategy. The flood season July to August is also the hunger period in rural Sierra Leone, and during this period some fish farmers harvest their brush parks, trap ponds and fish fences. Farmers could take advantage of this by increasing their fish supplies into the market and make high profit, which they could then use to buy the foodstuff they need and access high quality fish protein food.

\subsection{Relative Contributions of Different Livelihoods to Farmer's Incomes}

Differences in the relative contributions of different livelihoods to annual incomes of farmers in different regions of the country were mainly due to differences in prices of agricultural produce in the different parts of the country and in different seasons of the year [12]. Farmers, who were able to store their produce until prices were the highest due to food shortages, made more out of their farming activities than those who sold their produce at the harvesting period when everybody was harvesting and food was in abundance [13]. Similarly, some produce were commercially more important than others and farmers produce them for their market value, rather than consumptive values
[14].

The relatively low contribution of fish farming into farmers overall income could be attributed to the fact that many farmers interviewed were not producing much fish and that the bulk of the fish they produced was for home consumption. The few farmers who produced significant quantities of fish were harvesting their farms during the periods when fish was abundant in the markets and prices were low. These farmers were not able to preserve and store fish long enough to sell it at higher prices during periods of low fish supplies in the market.

Economic tree crops, like oil palm cocoa and coffee, on the other hand can be processed and stored by farmers and sold when market prices for such goods were the highest. Traditional fish farming therefore had high consumptive value to the farmers than income value relative to the other livelihoods activities. Farmers whose incomes were relatively high were therefore those who grew commercial crops, mined diamonds and gold or who were school teachers or retired civil servants.

\subsection{Summary of Livelihoods and Income Assessment}

All farmers employ the mixed strategy (multiple livelihoods) to meet their needs. In most cases, the primary livelihood strategy accounted for around half of their income. The choices of livelihoods the farmers made and the relative importance they attached to each of the livelihoods were based on the food value, income and social security provided by each. Fish farming has food, income and social security value potentials.

Generally, farmers were slightly better off in the South, although these results may be partially due to imbalance in those with paid employment. The equivalent income was around \$200 in the North and \$250 in the South; given that most farmers were the main income earner in a family/households. This indicates that per capita income for their families was probably typical for rural Sierra Leone [15]. 


\subsection{Cost of Farming Fish by Traditional Methods}

The bulk of fish production costs were on labour and cost of land. This two input cost items accounted for roughly $80 \%$ of the first year total production cost.

\subsubsection{Costs of Labour and Other Farm Inputs}

The most important cost item in the production system was labour, accounting for about $70 \%$ of the total production cost. The high cost of labour was due to the fact that a proxy rate of pay was used in the calculation and it was set as the daily rate for hiring labour. Rural farmers in Sierra Leone used simple home-made tools and implements. They cannot afford fertilizers and other agrochemicals. Pond construction is intensive labour and the informal labour market in the rural areas is such that apart from the payment to labour clubs to do work; they also have to be fed and this most often adds to the cost of labour significantly. However, most farmers interviewed in this assessment used unpaid family labour. It was therefore reasonable to assume that the actual labour cost of farming fish by traditional methods could be much lower than that predicted in this economic model, because the daily wage rate for labour clubs plus average amount required to feed labour club members were used in the calculations.

Cost of labour was higher in the North than in the South of the country (Table 6), accounting for $71 \%$ and $68 \%$ of total production costs, respectively. Higher cost of labour in the North may be due to the prevalence of labour clubs in the North, which has formalized the labour market in this region more compared to the South, where most of the farmers depended on family labour.

Cost of fingerlings on the other hand was higher in the South than in the North, accounting for $10 \%$ of total production cost in the North as compared to $15 \%$ in the South. Likely reason for the lower cost of fingerlings in the North was that there were more fish farms in the North than in the South and farmers were either buying fingerlings from other farmers or getting supply from the government hatchery at Makali; whereas there were fewer farms in the South and most farmers were obtaining their fish seed from the government experimental station in Bo town and transporting the fingerlings from the station to the farmers ponds, which may have added to the total cost of fingerlings.

The costs of other farm inputs, such as tools, feed and fertilizers, etc., were also recorded to be higher in the North than in the South. This was probably because the input supply sources in the North (Makeni and Magboraka) were far away from the farms in Tonkolili district and Bombali district, compared to farms in Bo district and Pujehun district, respectively.

\subsubsection{Cost of Land Rent}

Land rent was the 2nd highest input cost of fish farming. As with labour, a proxy value was used. The opportunity cost of using land for fish farming was considered as the forgone use value of the same plot of land. This was estimated as the value of crops (rice, vegetables and root tubers) that could have been grown where the ponds were. This is probably an overestimate, because it doesn't take into account the cost of production of the crops. However, the high estimate used in the analysis was justified, because the price of imported rice had gone up in Sierra Leone, due to inflation and market demand.

\subsection{Annual Costs and Net Incomes from Traditional Fish Farming}

Mean annual cost of fish farming was higher in the North than in the South of the country (Table 7). The most likely reason for this difference in the annual cost of production both for the first year production cycle and the recurrent expenditure is the scale of production. The cost per unit of output (kg of fish or ha of pond area) generally decrease with the increase in scale, as fixed costs are spread out over more units of output $^{3}$. Average pond size in the North was $375 \mathrm{~m}^{2}$, while the pond size in the South was $621 \mathrm{~m}^{2}$.

\footnotetext{
${ }^{3}$ http://study.com/academy/lesson/economies-of-scale-definitio n-benefits-examples.html.
} 
Although overall cost of fish production was higher in the North than in the South, profitability of the production system assessed turned out to be higher in the North than the South (Table 7). This was because that farmers in the North had smaller pond sizes, which allowed them easier options of harvesting fish at the request of customers even at middle of the rainy season when fish was most expensive. In the South, the pond sizes were very large, so requested sales harvest were difficult to manage in the rainy season. Farmers in the North therefore recorded higher fish sales to customers' request (\$802/ha/year in the North compared to $\$ 550 /$ ha/year in the South) (Table 6).

Mean annual income (in \$/ha/year) from fish farming decreased progressively with farm size, net profit (\$/ha/yr) were $\$ 1,496 \pm 80$ (small farms), $\$ 1,213 \pm 169$ (medium farms), $\$ 460 \pm 80$ (large farms) and $\$ 1,098 \pm 62$ (all farms). This could be explained by the large amount of labour input required to construct larger ponds (without proportional increase in the yield). This could have caused diminishing returns on labour ${ }^{4}$.

It was expected, based on the economics of scale principle, that the bigger ponds/farms would be a means of saving on land cost and labour, but the returns in this analysis were estimated based on actual money earned by selling fish harvested. It was recorded that the small ponds/farms were more profitable than the medium and large ones. The likely reasons for this were:

(1) The decrease in profit per unit surface area of ponds could be due to the complex relationship between supply and demand for fish in the village setting. Large farms who may have been producing more fish than the village market can buy in cash at particular times of the year. This could have forced fish farmers to sell their fish at lower than average prices, thus decreasing their total incomes;

(2) The farmers with large ponds were not getting

\footnotetext{
${ }^{4}$ http://www.investopedia.com/terms/l/lawofdiminishingmargin
} alreturn.asp. optimum stocking densities (because they lack the technical expertise to optimise stocking densities);

(3) The large ponds were more attractive to thieves/poachers and natural predators, leading to farmers recoding lower yields;

(4) Farmers with large ponds had more pressure or moral obligations to give away their fish freely to relatives, friends and important members of their communities.

The most likely reason for lower profitability from large ponds was that the farmers producing more fish than the village markets can profitably buy in cash, so the fish was either battered (exchanged for other goods), sold at below average prices, given on loan to friends and relatives or consumed by the farming households. Similarly, if the stocking densities were not optimized, farmers may have large ponds but very few fish in the ponds.

Generally, the annual net profit accrued by farmers appeared to be too small to justify fish farming as the only livelihood activities or occupation, especially when we consider that average farm size was less than 0.04 ha. However, if one considered that unpaid family labour was cost in the analysis, then it would appear that fish farming was making a meaningful contribution to household income and rural employment.

\subsection{Economic Viability of Traditional Methods of Fish} Farming

The study suggested that fish farming in Sierra Leone was economically viable for all the three farm size categories and regions. The small farms were more profitable than both medium and large farms. However, increasing sizes of ponds brought about savings on labour cost, which was most expensive cost item in the above production systems. The mean annual production cost (\$/ha/year) was $\$ 1,038 \pm 44$ (small farms), \$776 \pm 41 (medium farms), $\$ 560 \pm 64$ (large farms) and $\$ 819 \pm 44$ (all farms). The most labour-intensive activity (pond construction) was 
undertaken only once and total production costs in subsequent years (variable costs) were lower than total first year cost.

This decrease in the annual cost of production with increase in farm size may be related to the economics of scale of production. Economies of scale were the cost advantages that a producer obtains due to expansion. It was enjoyed by all farmers who expanded the scale of their operation as follows: (1) by hiring cheap labour to construct the ponds within a very short period; (2) by bulk buying of tools and implement; (3) by spreading the cost of land and other inputs over a greater range of output.

Larger scale production could also raise productivity and there was much more scope for growth through economies of scale in a developing country, such as Sierra Leone, than in larger industrialized countries [16].

\subsection{Benefit-Cost Ratio of Fish Farming}

The mean benefit-cost ratio of fish farming by traditional methods was very encouraging at 2.3 ; this means that the economic benefits of fish farming is more than double of its costs. Rice farming on the other hand which was ranked very highly by the farmers have been shown to have benefit-cost ratios of 0.59 for low yielding varieties, 0.78 for medium yielding rice varieties and 1.08 for high yielding rice varieties. The mean value for all the farmers output together was $0.83[17,18]$. Fish farming by traditional methods therefore offers greater economic benefit than rice farming.

\subsection{Payback Period}

Some farmers had access to loans from informal financial institutions, such as the "Osusu", without interest, they would be able to pay back their loans in 4-6 years. This was encouraging, because cash crops (cocoa and coffee) and oil palm plantations which were regarded by farmers to be more rewarding, and provided rural farmers social security benefits have waiting periods of at least five years, during which the farmers continuously invest on their production without harvesting because the trees would not have reached maturity and started producing.

The payback periods obtained for both interest free loans and at $20 \%$ interest rates were also attractive in that if farmers had access to loans for the formal banking system, they would be able to pay back the loans within a reasonable period of approximately seven years. This payback period was calculated for low technology production systems. If modern highly productive technologies were used and natural predation and theft were controlled or minimized by the farmers, the payback period could be less than the minimum of five year waiting period for tree crops perceived by many farmers to be more productive and rewarding farming system in rural Sierra Leone.

Currently, subsistent farmers in rural Sierra Leone only have access to loans and credit facilities through informal financial arrangements, such as "Osusu", and village money lenders/traders. The amount of money available from these sources was small relative to that needed for large scale investment into aquaculture production. Subsistent traditional fish farmers in this study currently eat most of the fish they produce, and the estimated incomes in this analysis was actually farmer's savings on animal protein food for their households and some form of "social capital" savings.

The higher value of the payback period for the large farms indicated that farmers with large farms were investing more on labour with minimum increase in the level of production, in other words, the law of diminishing returns on labour could be affecting these production systems. Beyond some point, each additional unit of variable input would yield less output. If one factor of production was increased (in this case labour), while the others remained constant, the overall returns would decrease after a certain point. Each additional labourer would add relatively less output, because he has less and less of the fixed amount of land to work [19]. 


\section{Conclusions}

Ranking of local people’s livelihoods was based on a combination of criteria (food, income and social security values). Household consumption value of livelihoods was the most important, so rice farming was considered the most important production system and fish farming ranked the 5th out of 19 different livelihoods recorded in the study. Fish farming can be made more attractive to rural farmers in Sierra Leone, if it could be reinterpreted as an income generating activity, like tree crops rather than a minor subsistence activity, or if introduced as integrated farming system with their favourite rice farming.

The economic evaluation of fish farming in ponds using proxy measures for the cost of labour and land was economically viable (profitable production system). However, the level of profitability recorded in this analysis was not perceived as such by local farmers who were engaged in fish farming. Possible reasons for fish farmer's negative perception of fish farming were:

(1) The income estimated from the fish production systems in the analysis was not all realized in cash by the farmers. More than one-quarter (27\%) of total gross income recorded in the study was an estimated value of farmed fish consumed by farmers. An additional $8 \%$ of income was an estimated value for fish used by farmers to restock ponds for subsequent production cycles;

(2) The $44 \%$ of total gross income reported by farmers at the main harvest was also not always in cash. Some farmers were reported to exchange their fish (batter) for rice, cassava, palm oil, magi, etc.;

(3) Farmers in rural Sierra Leone did not attach actual money value to labour and the food they ate, it was also likely that they did not perceive the fish eaten in their households as income in monetary terms coming from fish farming;

(4) Farmers were giving away significant quantities of their farmed fish freely to friends, relatives, chiefs, religious leaders, etc. (investing on “social capital”).
The value of fish given away was estimated as total gross income accruing to farmers, but the farmers did not receive that as cash.

If the analysis included intangible benefits that farmers received from fish farming, such as "social capital” and "kinship", which could later yield indirect benefits to the farmer, such as free labour in future work, protection from chiefs if the farmer has a problem in the village and support from the chief, then fish farming could be seen as a very profitable investment in both monetary and social terms.

Incomes received from traditional fish farming by rural farmers was relatively lower than that received from other livelihoods activities, because relatively lower priority and effort were devoted to fish farming; the scale of production and technologies used may be responsible for the low income levels from fish farming.

It was therefore concluded that fish farmers in Sierra Leone have a very clear and very consistent set of priorities; aquaculture has to fit within those priorities. Fish farming was the 5th in a line-up of 19 important livelihoods activities. There was only a relatively short period when farmers have free time and have sufficient food (late January to early March) to construct and manage ponds, and it was recorded that during this period the farmers were also often busy with other activities (social events, such marriages, circumcisions, etc.). However, farmers recognized that tree crops provided long-term benefits after they have been established, so that fish ponds could be seen in a similar manner or even better because farmers could start getting returns from fish farming in less than one year of establishing the farm; whereas, they have to wait for a minimum period of five years before they could get returns from most tree crops. Similarly, fish farming has been shown to be more rewarding than rice farming.

The opportunity cost of farmers growing fish was rice farming, and farmers were not ready to give up rice farming for fish farming easily, as rice was their 
staple food, and the level of fish production and market value for fish was not sufficient to compensate for the cost of rice they would loose if they devoted more of their time on fish farming.

\section{References}

[1] FAO. 2008. Report of the Expert Consultation on the Assessment of Socio-Economic Impacts of Aquaculture. FAO Fisheries Report No. 861, FAO, Rome.

[2] Frankic, A., and Hershner, C. 2003. "Sustainable Aquaculture: Developing the Promise of Aquaculture.” Aquaculture International 11 (6): 517-30.

[3] World Bank. 2005. Managing Agricultural Production Risk: Innovations in Developing Countries. Report No. 32727-GLB, the World Bank Agriculture and Rural Development Department.

[4] Taylor, J. E., and Adelman, I. 1996. Village Economies: The Design, Estimation and Use of Village Wide Economic Models. Cambridge: Cambridge University Press.

[5] Sankoh, S. K. 2009. "Aquiculture in Sierra Leone: Traditional Systems and Prospects for Market Oriented Development.” Ph.D. thesis, Faculty of Natural Sciences, Aquaculture Systems Group, Institute of Aquaculture, University of Stirling, Stirling, UK.

[6] Sankoh, S. K. 2005. Report of the Rapid Rural and Participatory Rural Appraisals (RRA/PRAs) for the Verification of Data Generated in the National Aquaculture Baseline Survey.

[7] Statistics Sierra Leone. 2004. Statistics Sierra Leone: Annual Statistical Digest 2004.

[8] Spencer, D. S. C., and Wilson, S. D. C. 2009. Economics of Rice Production in Sierra Leone. Report of a Survey in Three Northern Districts, June 25, 2009.

[9] Conteh, A. M. H., Yan, X., and Sankoh, F. P. 2002. "The Influence of Price on Rice Production in Sierra Leone.”
Agricultural Sciences 3 (4): 462-9.

[10] Spencer, D. S. C. 2009. Farmer Perceptions, Child Labour and Economics of Tree Crops Production and Marketing in Kailahun, Kenema and Kono Districts of Sierra Leone. Report of the Promoting Agriculture, Governance and the Environment (PAGE) Project.

[11] Sanni, L. O., Onadipe, O. O., Llona, P., Mussagy, M. D., Abass, A., and Dixon, A. G. O. 2009. Successes and Challenges of Cassava Enterprises in West Africa: A Case Study of Nigeria, Bénin and Sierra Leone. Ibadan, Nigeria: IITA.

[12] Statistics Sierra Leone. 2008. Statistics Sierra Leone: Annual Statistical Digest 2005/2006.

[13] Statistics Sierra Leone. 2013. A Poverty Profile for Sierra Leone. The World Bank Poverty Reduction and Economic Management Unit for Africa.

[14] Sinclair, F. L., and Joshi, L. 2000. "Taking Local Knowledge about Trees Seriously.” In Forestry, Forest Users and Research: New Ways of Learning, edited by Lawrence, A. Wageningen, Netherlands: European Tropical Forest Research Network, 45-61.

[15] GOSL. 2005. Sierra Leone: Poverty Reduction Strategy Paper (SL-PRSP). IMF Country Report No. 05/191, A National Programme for Food Security, Job Creation and Good Governance (2005-2007).

[16] Pingali, P. L., and Rosegrant, M. W. 1995. “Agricultural Commercialization and Diversification: Processes and Policies.” Food Policy 20 (3): 171-85.

[17] FAO. 2001. Agricultural Investment and Productivity in Developing Countries, edited by Zepeda, L. UN: FAO Economic and Social Development.

[18] Karr, G. L., Njoku, O. A., and Kallon, M. F. 1972. "Economics of the Upland and the Inland Valley Swamp Rice Production Systems in Sierra Leone.” Illinois Agricultural Economics 12 (1): 12-7.

[19] Stanlake, G. F., and Grant, S. J. 1995. Introductory Economics, 6th ed.. Cardiff, United Kingdom: Longman Group Limitted, 538. 